\title{
Educación para el emprendimiento: análisis y aportes a la política pública de educación en Colombia desde la sistematización de experiencias en instituciones de educación básica y media ${ }^{1}$
}

\author{
Claudia Marcela Guarnizo Vargas ${ }^{2}$, Jairo Rodrigo Velásquez Moreno ${ }^{3}$, Claudia Patricia \\ Jiménez Guzmán ${ }^{4}$ Beatriz Helena Álzate Gómez ${ }^{5}$
}

Recibido: septiembre 2017 / Evaluado: Diciembre 2017 / Aceptado: Enero 2017

Resumen. La educación para el emprendimiento funciona como dispositivo gubernamental en instituciones educativas de educación básica y media de Colombia desde el 2006 en el marco de la ley 1014 y desde ahí, se han construido diversas interpretaciones que se traducen en discursos, los cuales orientan prácticas, que en este proyecto se han recontextualizado y vale la pena analizarlas en clave de aportes a la política pública educativa. El objetivo es divulgar nuevos conocimientos de las mismas instituciones participantes en la sistematización de experiencias relacionadas con la cultura del emprendimiento. El método utilizado se enmarca en una investigación cualitativa centrada en la sistematización de experiencias y el posterior análisis de enunciados en los diversos relatos. Como resultado se observaron prácticas institucionales que movilizan la cultura institucional para el emprendimiento y la empresarialidad (CIEE); procesos de gestión institucional que empoderan a las personas en la línea de una educación centrada en la formación integral que responda a los retos del contexto actual. Como conclusiones de este trabajo se evidencia que la política de educación colombiana puede ser fortalecida por las reflexiones y prácticas institucionales que han asumido que la educación para el emprendimiento debe tener una concepción de cultura institucional pertinente para los territorios.

Palabras clave: gestión del centro de enseñanza; cambio de actitud; política de la educación; emprendimiento.

1 Este artículo hace parte del proyecto denominado "Maestros y maestras como productores de saberes y conocimientos alrededor de la Cultura Institucional para el emprendimiento y la empresarialidad (CIEE)", adscrito a los grupos de investigación: Educación y Pedagogía: Saberes Imaginarios e intersubjetividades de la Universidad de Manizales y Grupo interdisciplinario de investigación en pedagogía para la innovación y el desarrollo Unicafam de la Fundación Universitaria Cafam en Colombia. Fue financiado por la alianza de la Fundación Universitaria Cafam, la Universidad de Manizales y la Central Hidroeléctrica de Caldas CHEC con el código: EP/2015-1-001.

2 Fundación Universitaria Cafam (Bogotá, Colombia)

E-mail: marcela.guarnizo@unicafam.edu.co

3 Fundación Universitaria Cafam (Bogotá, Colombia)

E-mail:jairo.velasquez@unicafam.edu.co

4 Universidad de Manizales (Manizales-Colombia).

E-mail: p.jimenez@umanizales.edu.co

5 Universidad de Manizales (Manizales-Colombia).

E-mail: bettya@umanizales.edu.co 
[en] Education for the entrepreneurship: analysis and contributions to the public policy of education in Colombia from the systematization of experiences in institutions of basic and secondary education

\begin{abstract}
The Entrepreneurship education has functioned as a governmental device in institutions of primary and secondary education in Colombia from 2006 under the law 1014 and from there, different interpretations have been constructed that translate into speeches, which guide practices, which in this project have been recontextualized and worth analyzing in key contributions to public educational policy. The objective is to disseminate new knowledge of the same participating institutions in the systematization of experiences related to entrepreneurship culture. The method used is framed on a research qualitative approach focused on the systematization of experiences and the subsequent analysis of statements in the various stories (narrations). As a result, institutional practices that mobilized the institutional culture for entrepreneurship were observed; Institutional management processes that empower people along the lines of an education focused on comprehensive training that responds to the challenges in the current context. As conclusions of this work it is evident that the Colombian education policy can be strengthened by the reflections and institutional practices that have assumed that education for entrepreneurship must have a conception of institutional culture relevant to the territories.
\end{abstract}

Keywords: management of the teaching center; change of attitude; education policy, entrepreneurship.

Sumario. 1. Introducción. 2. Método. 3. Búsqueda de información. 4. Análisis de la información. 5. Resultados. 6. Discusión. 7. Conclusiones. 8. Agradecimientos. 9. Referencias bibliográficas.

Cómo citar: Guarnizo Vargas, C. M; Velásquez Moreno, J. R.; Jiménez Guzmán, C. P.; Álzate Gómez, B. H. (2019). Educación para el emprendimiento: análisis y aportes a la política pública de educación en Colombia desde la sistematización de experiencias en instituciones de educación básica y media. Revista Complutense de Educación, 30(1), 225-243.

\title{
1. Introducción
}

En Colombia, desde hace más de diez años, se viene reflexionando sobre la forma de lograr que la educación de niños y jóvenes los prepare para enfrentar el mundo productivo, desde un enfoque de formación de competencias, entre ellas, las laborales generales, entendidas según (MEN, 2006, pág. 5) como todos “(...) aquellos conocimientos, habilidades y actitudes, que son necesarios para que los jóvenes se desempeñen con eficiencia como seres productivos". En este tipo de competencias se encuentran las relacionadas con el emprendimiento y la empresarialidad. Este es un concepto que trasciende lo económico y se proyecta a otros ámbitos de desarrollo de la vida familiar, laboral, social y personal.

Se entiende el emprendimiento como,

Una manera de pensar y actuar orientada hacia la creación de riqueza centrada en las oportunidades, planteada con visión global y llevada a cabo mediante un liderazgo equilibrado y la gestión de un riesgo calculado, su resultado es la creación de valor que beneficia a la empresa, la economía y la sociedad; y la empresarialidad se entiende como el despliegue de la capacidad creativa de la persona sobre la realidad que le rodea para percibir e interrelacionarse con su entorno, mediando para ello las competencias empresariales (Ley 1014, 2006, pág. 1) 
El anterior es el concepto que está en la ley y que dio origen en enero de 2006 a ésta discusión sobre la articulación del emprendimiento en la educación preescolar, básica y media en Colombia.

Ésta establece en su artículo 13 que las instituciones educativas deben definir un área específica de formación para el emprendimiento y la generación de empresas, que debe incorporarse al currículo y desarrollarse en el plan de estudios. Esta área debe ser complementada con actividades institucionales que permitan resaltar el trabajo de los estudiantes, como ferias empresariales, diseñar y desarrollar módulos de formación para el emprendimiento que sean la cátedra empresarial. El espíritu de estas acciones es "capacitar al estudiante en el desarrollo de capacidades emprendedoras para generar empresas con una visión clara de su entorno que le permita asumir retos y responsabilidades" (Ley 1014, 2006, págs. 6-7)

Como respuesta a esta ley, las instituciones diseñaron e implementaron una cátedra o una nueva asignatura de educación para el emprendimiento, que desde sexto grado, y en algunos casos desde preescolar, desarrolla un proceso de formación orientado a la creación de empresas.

En 2012, el Ministerio de Educación Nacional de Colombia, a partir del análisis y la interpretación de los conceptos de emprendimiento y empresarialidad en contraste con las prácticas institucionales lidera un proceso de resignificación que oriente los currículos y permita el desarrollo de un enfoque de educación para el emprendimiento más integral y articulado a la gestión institucional y al desarrollo tanto de las competencias básicas como ciudadanas en las instituciones educativas.

Entonces, publica la Guía 39 (MEN, 2012) que ha propiciado que entidades territoriales e instituciones desarrollen proyectos para el fomento de la cultura para el emprendimiento, y el desarrollo de actitudes emprendedoras, conceptos entendidos como:

Cultura del emprendimiento:(...) un conjunto de valores, creencias, ideologías, hábitos, costumbres y normas, que comparten los individuos en la organización y que surgen de la interrelación social, los cuales generan patrones de comportamiento colectivos que establece una identidad entre sus miembros y los identifica de otra organización. (Ley 1014 de 2006, art. $1^{\circ}$ ).

Actitudes emprendedoras: "Se entiende por la disposición personal a actuar de forma proactiva frente a cualquier situación de la vida. Esta actitud genera ideas innovadoras que pueden materializarse en proyecto y alternativas para la satisfacción de necesidades y solución de problemáticas. Así mismo propicia el crecimiento y la mejora permanente del proyecto de vida. (MEN, 2012, pág. 12)

Enfoque que se puede contrastar y reafirmar en los esfuerzos que hacen otros países, por ejemplo en la unión europea le apuestan a una educación que incentive la formación de unas competencias claves para el aprendizaje permanente, entre las cuales señalan las del sentido de iniciativa y el espíritu emprendedor, centradas en la formación de actitudes favorables para emprender, como son, tener iniciativa, capacidad de asumir riesgos y de manejar la incertidumbre, asuntos que se aplican a los retos del mundo productivo actual y que en el presente estudio son un punto recurrente en el enfoque del emprendimiento institucional (Ministerio de Educación, cultura y deporte, 2015, págs. 17-20) 
Particularmente en España, se plantea la necesidad de profundizar en temas de educación para el emprendimiento mediante la formación de actitudes que movilicen el comportamiento emprendedor, asunto que establece una línea base para orientar los procesos educativos en la educación secundaria y media, tal como lo señalan (Oliver, Galiana, \& Gutiérrez, 2016, pág. 184) cuando encuentran que en España es importante adelantar estudios que midan la actitud emprendedora para generar una cultura favorable al emprendimiento, asunto clave en el proyecto que se desarrolló en torno a la CIEE y que es el centro de ésta investigación.

En Colombia, éste enfoque integral se empezó a implementar en las instituciones de educación básica y media, entre éstas, se encuentran las de Caldas y Risaralda, dos departamentos colombianos que, gracias al apoyo de las empresas y las Secretarías de Educación, son los más sostenibles, razón por la cual son el centro de análisis de esta investigación.

Este camino que han tenido que recorrer las instituciones en el país ha sido complementado y fortalecido específicamente en Caldas y Risaralda con el proceso de formación, asesoría y acompañamiento que la Fundación Universitaria Cafam (Unicafam) ha desarrollado en convenio con la Central Hidroeléctrica de Caldas-CHECGrupo epm, las secretarias de educación y la Universidad de Manizales, razón por la cual se ha logrado fortalecimiento curricular y cambios institucionales significativos, que ameritan de un análisis profundo para comprender cómo desde estas experiencias se ha logrado llevar a la práctica y trascender los orientaciones establecidas en la política educativa.

En este sentido, la pregunta de la investigación que inspiró éste ejercicio fue: ¿Qué aportes brindan las experiencias en torno a los procesos de enseñanza y de aprendizaje del emprendimiento en los establecimientos educativos públicos de educación básica y media, a la política pública educativa actual?

Es un trabajo que tiene como objetivo divulgar nuevo conocimiento basado en el análisis que surge de las prácticas institucionales centradas en la CIEE y que sucede en instituciones de Caldas y Risaralda. Todo con el fin de fortalecer y reorientar procesos de formación y fomento de la cultura del emprendimiento que se vienen desarrollando en el país bajo la orientación de varios actores, entidades y redes de emprendimiento a nivel nacional y territorial, de tal manera que permitan el enriquecimiento de la política pública de educación en el país.

\section{Método}

\section{Diseño del método de estudio}

Es una investigación cualitativa desarrollada mediante la sistematización de experiencias que de acuerdo con (Sampieri, Fernández, \& Baptista, 2010, págs. 7-9), se considera cualitativa porque se caracteriza por ser una metodología que no fija previamente unas variables de estudio sino que al contrario se hace un proceso de inmersión en el cual van emergiendo interpretaciones y múltiples preguntas que requieren ser analizadas; algunos autores consideran éste método como una "investigación de segundo orden", que genera procesos de auto referencia y auto observación, y utiliza lógicas diversas y nuevas comprensiones de la realidad buscando producir nuevo conocimiento (Molina, 2001, pág. 21) 


\section{Escenario}

Se lleva a cabo en el marco del proyecto de formación y asesoría desarrollado por Unicafam y la CHEC en los departamentos de Caldas y Risaralda (Colombia), entre los años 2015 y 2016.

\section{Población y muestra}

La investigación se desarrolló con los productos de 12 instituciones educativas que participaron en la sistematización de experiencias (Tabla 1), incluyendo los aportes de la profesional que dinamizó el proceso en la región. Es de aclarar que las instituciones que participaron en el proceso de formación fueron invitadas a participar en la sistematización y de manera voluntaria aceptaron 10 instituciones educativas y 2 categorizadas como Escuela Normal Superior, instituciones en las cuales se forman maestros y maestras en el país.

Tabla 1. Instituciones educativas participantes.

\begin{tabular}{|c|c|c|c|}
\hline No. & Institución educativa & Municipio & Departamento \\
\hline 1 & IE Marillac & Santa Rosa de Cabal & Risaralda \\
\hline 2 & I.E. Aureliano Flórez & Anserma & \multirow{10}{*}{ Caldas } \\
\hline 3 & I.E. Santa Teresita & \multirow{3}{*}{ Chinchiná } & \\
\hline 4 & IE San Francisco de Paula & & \\
\hline 5 & IE Santo Domingo Savio & & \\
\hline 6 & IE Riosucio & \multirow[b]{2}{*}{ Riosucio } & \\
\hline 7 & $\begin{array}{c}\text { Escuela Normal Superior (ENS) } \\
\text { Sagrado Corazón }\end{array}$ & & \\
\hline 8 & ENS La Candelaria & \multirow{2}{*}{ Marquetalia } & \\
\hline 9 & IE Juan XXIII & & \\
\hline 10 & IE Pio XI & Aranzazu & \\
\hline 11 & IE Intec & Supía & \\
\hline 12 & IE San Jorge & Manizales & Manizales \\
\hline
\end{tabular}

\section{Búsqueda de información}

El relato, como instrumento en el cual, a través de la narrativa, se sistematizó la experiencia, se construyó en dos momentos, el primero tiene a la práctica como el primer referente, y parte de la pregunta como motivadora del proceso, lo que permitió construir una primera descripción. El segundo retoma el ejercicio anterior y se plantea nuevas preguntas, con una mirada crítica y la construcción de un nuevo relato mediado por un conjunto de procesos y herramientas. 
Los procesos se relacionan con ejercicios de trabajo centrado en un equipo de sistematización, alrededor del cual se desarrolla un diálogo de saberes y una negociación cultural, acompañada de la reflexión y la identificación de líneas de fuerza que caracterizaran el análisis de la experiencia. Los segundos instrumentos son propios de la investigación cualitativa como el cuaderno de notas y los diarios de campo. Estos fueron la fuente de información y recolección de datos de referencia para realizar un proceso de reconstrucción y análisis de las experiencias que trascendió el nivel descriptivo hasta llegar a un nivel más avanzado de interpretación y reflexión teórica, con el propósito de producir nuevo conocimiento relacionado con el fomento de la CIEE que emerge de la práctica.

De acuerdo con los elementos que han caracterizado al proyecto, se trazó una ruta metodológica con un enfoque particular para la sistematización de experiencias, que guarda relación con la propuesta de (Mejía, 2012) y que se detalla en el siguiente esquema, ver gráfica 1:

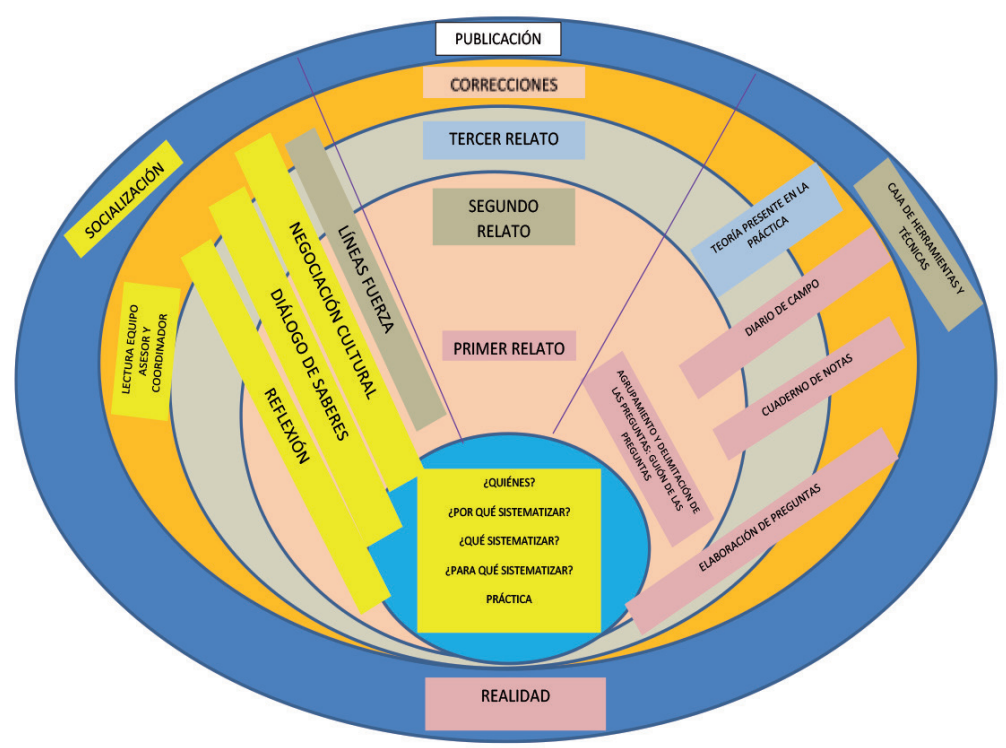

Gráfica 1. Ruta de sistematización de experiencias en EE de Caldas y Risaralda Fuente: elaboración de los autores.

Como producto de este proceso, se obtuvieron relatos de los participantes, que luego, mediante técnicas de análisis cualitativo, fueron interpretados y analizados para responder a cada una de las preguntas planteadas y emergentes durante el proceso.

El análisis de los relatos se centró en identificar cómo han contribuido las experiencias a enriquecer la política educativa actual, para lo cual se tomaron como referencia los elementos centrales establecidos en el plan de desarrollo "Todos por un nuevo país" (DNP, 2014) (Tabla 2). De este planteamiento de política nacional se tomaron las categorías iniciales de análisis de ésta investigación y que se presentan a continuación: 
Tabla 2. Categorías de análisis según la política educativa de Colombia 2014-2018.

\begin{tabular}{|c|}
\hline Categoría inicial de análisis \\
\hline Resultados en pruebas externas \\
\hline Retos de la globalización \\
\hline Competencias ciudadanas en el marco de la paz \\
\hline Sujetos emprendedores \\
\hline Cualificación del personal docente y de las prácticas pedagógicas \\
\hline
\end{tabular}

\section{Análisis de la información}

El análisis se centró en la interpretación de los enunciados de las instituciones participantes extraídos de sus relatos, para identificar aspectos claves y novedosos, que muestran empoderamiento de un enfoque integral de educación para el emprendimiento. Se entiende los enunciados como un acontecimiento arqueológico, los cuales según (Foucault, 2004) citado por (Velásquez \& Zamudio, 2012, pág. 135) no se centra en las continuidades sino en las discontinuidades y se prioriza el contexto en el que se desarrolla, (...) es un acontecimiento extraño, indudablemente: en primer lugar porque está ligado por una parte a un gesto de escritura o a la articulación de una palabra, pero que por otra se abre a sí mismo una existencia remanente en el campo de una memoria.

Es un ejercicio que visibiliza desde una concepción institucional, una interpretación de una educación para el emprendimiento que se convierte en dispositivos emergentes de subjetivación, los cuales, según Deleuze citado por (Dallorso, 2012, pág. 52) se ubican en la "cuarta línea del dispositivo", según la cual, se propician nuevas concepciones e interpretaciones que subvierten el principio establecido.

Son nuevas interpretaciones que las instituciones han construido y han sido la base para producir nuevas formas de pensar y actuar, que indican procesos de transformación y empoderamiento institucional alternativo entre lo que plantean los discursos gubernamentales y lo conciben las instituciones educativas.

En el análisis se identificaron 104 enunciados de las instituciones participantes incluido el relato de la dinamizadora regional del proceso. El diseño de la investigación fue aprobado por los comités de investigación de las Universidades de Manizales y la Fundación Universitaria Cafam respectivamente.

\section{Resultados}

Después de analizar los relatos, se encontraron algunas categorías emergentes que complementan a las establecidas por la política pública educativa y que permiten ampliar el análisis e identificar otros aspectos que pueden enriquecer la relación que se pretende establecer entre la experiencia vivida y los planteamientos de dicha política. Estas categorías fueron: 
Tabla 3. Categorías emergentes del análisis de relatos.

\begin{tabular}{|c|c|}
\hline Categorías emergentes & Interpretación desde lo enunciado por las instituciones \\
\hline $\begin{array}{l}\text { Direccionamiento estratégico de } \\
\text { las instituciones como factor de } \\
\text { calidad }\end{array}$ & $\begin{array}{l}\text { El direccionamiento estratégico y la calidad son conceptos } \\
\text { muy asociados a las dinámicas de gestión institucional, que } \\
\text { convocan a todos los integrantes de una organización a que } \\
\text { reconozcan y comprendan el horizonte institucional y actúen } \\
\text { de manera consecuente con sus principios, su visión y su } \\
\text { misión. }\end{array}$ \\
\hline $\begin{array}{l}\text { Cambio de paradigma en las } \\
\text { dinámicas institucionales hacia } \\
\text { una educación pertinente para los } \\
\text { territorios }\end{array}$ & $\begin{array}{l}\text { Hace referencia a cambios en la forma de pensar, sentir } \\
\text { y actuar de los miembros de la comunidad educativa en } \\
\text { relación a los propósitos educativos, centrándose en una } \\
\text { educación para el emprendimiento que atienda a necesidades } \\
\text { institucionales y del entorno. }\end{array}$ \\
\hline $\begin{array}{l}\text { Rendición de cuentas y trabajo } \\
\text { colaborativo en las instituciones } \\
\text { educativas }\end{array}$ & $\begin{array}{l}\text { Cuando se fomenta la CIEE en las instituciones, se genera una } \\
\text { reflexión sobre lo que hacen las organizaciones educativas, } \\
\text { los logros obtenidos, el balance de las acciones en la línea de } \\
\text { desarrollar un proceso de transparencia y autorreflexión que } \\
\text { fortalezca la comunidad y el trabajo colaborativo hacia un } \\
\text { propósito común. }\end{array}$ \\
\hline $\begin{array}{l}\text { Sinergia institucional e } \\
\text { interinstitucional }\end{array}$ & $\begin{array}{l}\text { Un elemento clave para cohesionar equipos de trabajo es la } \\
\text { articulación entre varios actores de la comunidad educativa y } \\
\text { con aliados estratégicos que pueden fortalecer el proceso. }\end{array}$ \\
\hline $\begin{array}{l}\text { Propuestas de valor en las } \\
\text { instituciones }\end{array}$ & $\begin{array}{l}\text { Cuando las instituciones generan acciones complementarias y } \\
\text { diferenciadoras establecen un sello particular del proceso que } \\
\text { genera compromiso y participación }\end{array}$ \\
\hline $\begin{array}{l}\text { Compromiso y transformación } \\
\text { institucional - sostenibilidad }\end{array}$ & $\begin{array}{l}\text { El trabajo en torno a la CIEE genera gran compromiso de los } \\
\text { miembros de la comunidad educativa para que los propósitos } \\
\text { y las prácticas de una educación para el emprendimiento se } \\
\text { consoliden y generen cambios, sostenibilidad y proyección. }\end{array}$ \\
\hline
\end{tabular}

El análisis se realizó mediante matrices construidas con los enunciados identificados en los documentos institucionales que resultaron de la sistematización de experiencias, como resultado de éste proceso se observa que algunos enunciados se manifiestan con regularidad en dos de las once categorías: un 59\% de los enunciados relacionan la categoría "Cualificación del personal docente y de las prácticas pedagógicas”, ver gráfica 2:

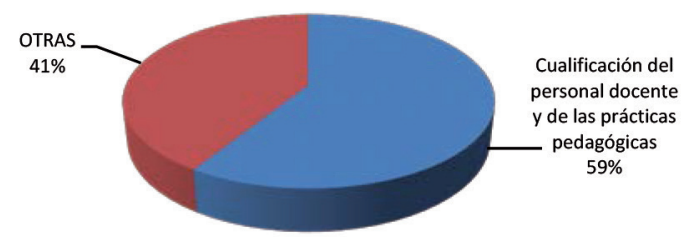

Gráfica 2. Cualificación del personal docente y de las prácticas pedagógicas. 
En su orden y con un $45 \%$ aparece la categoría: "Direccionamiento estratégico de las instituciones como factor de calidad" ver gráfica 3:

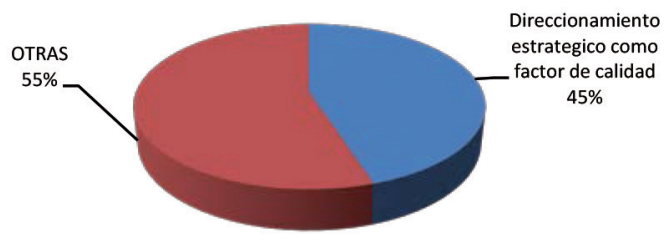

Gráfica 3. Direccionamiento estratégico de las instituciones como factor de calidad.

Siguen 2 categorías en las que coincidieron varios enunciados institucionales (gráficas 4 y 5). Con un 23\% las categorías "competencias ciudadanas en el marco de la paz" " "Cambio de paradigma en las dinámicas institucionales hacia una educación pertinente para los territorios" respectivamente:

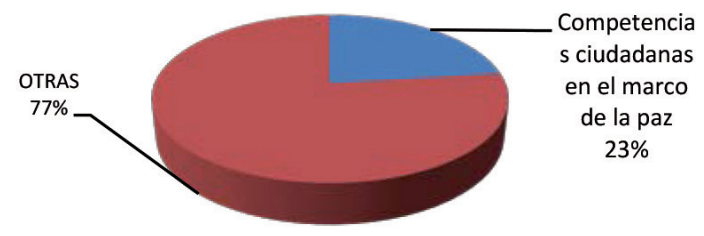

Gráfica 4. Competencias ciudadanas en el marco de la paz.

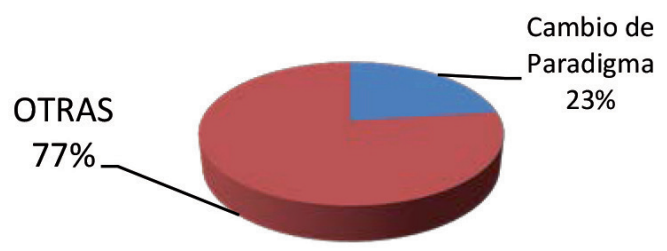

Gráfica 5. Cambio de paradigma en las dinámicas institucionales hacia una educación pertinente para los territorios.

Con un $21 \%$ la categoría "Sujetos emprendedores" ver gráfica 6 :

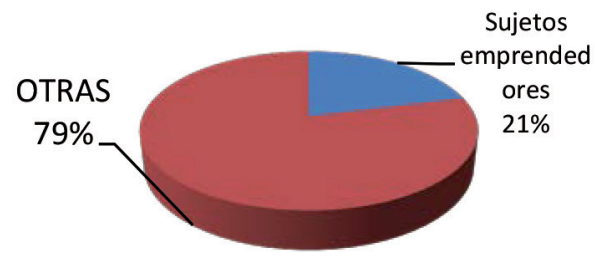

Gráfica 6. Sujetos emprendedores. 
Y con un 18\% la categoría "Retos de la globalización", ver gráfica 7.

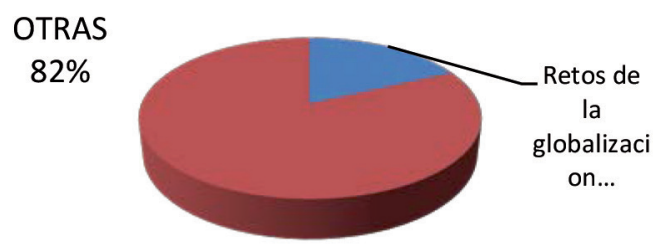

Gráfica 7. Retos de la Globalización.

Teniendo en cuenta los hallazgos más relevantes, y para efectos del análisis, se agruparon las categorías más generales en 3 macro categorías que engloban los principales aportes de los relatos de las instituciones, son ellas las prácticas que movilizan; la gestión que empodera; y de vuelta al sujeto, resultados que se exponen a continuación.

\section{Las prácticas que movilizan}

La orientación del proceso académico en las instituciones reúne varias concepciones que orientan las prácticas curriculares, incluyendo las que se ubican en el direccionamiento de los principios orientadores, la relación con modelos pedagógicos y planes de clase.

En esta sistematización, se observan varios enfoques que las instituciones exponen como escenarios propicios para que la educación para el emprendimiento se articule con la vida institucional y con los procesos formativos de algunas áreas del conocimiento y proyectos pedagógicos. En la tabla 4, se presentan los principales hallazgos.

Tabla 4. Enunciados con prácticas movilizadoras de la CIEE.

\begin{tabular}{|c|l|}
\hline \multicolumn{1}{|c|}{ Actor } & \multicolumn{1}{c|}{ Enunciado } \\
\hline ENS Sagrado Corazón & $\begin{array}{l}\text { El Emprendimiento... se entiende según el modelo Socio } \\
\text { Humanista Problematizante como la respuesta a la creación de } \\
\text { ambientes especiales que brinda la escuela, para que el estudiante } \\
\text { con lo aprendido en el aula y lo que encuentra en el contexto, lo } \\
\text { transforme y formule soluciones adecuadas de manera asertiva, en } \\
\text { función de una convivencia armónica con el medio. }\end{array}$ \\
\hline ENS La Candelaria & $\begin{array}{l}\text { La pedagogía dialogante al interesarse por formar un hombre y } \\
\text { una mujer en lo afectivo, cognitivo y praxeológico a través de } \\
\text { una relación interestructurante que reivindica el rol del maestro y } \\
\text { del estudiante en el acto pedagógico... da vía libre a las actitudes } \\
\text { emprendedoras como una conjunción de estas tres dimensiones } \\
\text { que sitúa al sujeto de cara al mundo, a las oportunidades y a su } \\
\text { proyecto de vida individual y social. }\end{array}$ \\
\hline
\end{tabular}




\begin{tabular}{|c|l|}
\hline \multicolumn{1}{|c|}{ Actor } & \multicolumn{1}{c|}{ Enunciado } \\
\hline IE Santo Domingo Savio & $\begin{array}{l}\text { En el diagnóstico con la comunidad educativa en mesas de } \\
\text { trabajo se concluyó que todas las actividades planteadas } \\
\text { en los programas, planes y proyectos formulados en el } \\
\text { proyecto educativo institucional (PEI) fortalecían actitudes } \\
\text { emprendedoras pero en forma aislada, sin cubrir toda la } \\
\text { institución, y no estaban intencionadas para tal fin en todas las } \\
\text { áreas del conocimiento. }\end{array}$ \\
\hline IE Juan XXIII & $\begin{array}{l}\text { El equipo líder de emprendimiento, que timonea el navío hace } \\
\text { las respectivas orientaciones para construir rúbricas de muchos } \\
\text { eventos pedagógicos y de conocimiento que se dan en la formación } \\
\text { y en ellas debía presentarse ampliamente la intención del } \\
\text { emprendimiento ambiental, es así como este penetra constantemente } \\
\text { cada acto escolar. }\end{array}$ \\
\hline IE San Jorge & $\begin{array}{l}\text { En la institución, se vivencia la metodología de Escuela Activa } \\
\text { Urbana fundamentada en el trabajo y la reflexión de todos los } \\
\text { pedagogos que quisieron vivenciar la educación de una manera } \\
\text { diferente... siendo esta un escenario propicio para el desarrollo de } \\
\text { actitudes emprendedoras. }\end{array}$ \\
\hline IE Pio XI & $\begin{array}{l}\text { La asignatura del Emprendimiento hacía parte de la malla curricular } \\
\text { en las áreas optativas que el estudiante debía desarrollar. Hoy, el } \\
\text { emprendimiento es transversal a todas las áreas y se desarrolla } \\
\text { desde preescolar. }\end{array}$ \\
\hline $\begin{array}{l}\text { La Institución educativa Pío XI considera la cultura del } \\
\text { emprendimiento y la empresarialidad como pilar de reorganización } \\
\text { de procesos institucionales, colectivos y personales que no solo } \\
\text { generan transformaciones sino que también incide en la conciencia } \\
\text { colectiva hacia procesos organizacionales y cambios que esta } \\
\text { cultura ha generado en la IE. }\end{array}$ \\
\hline
\end{tabular}

\section{La gestión que empodera}

El concepto de gestión en las instituciones sitúa la acción de hacer y de emprender con sentido, con proyección y perspectiva. En este sentido, la experiencia de las instituciones indica la interiorización de un principio que se trabajó en los espacios de formación: "La cultura del emprendimiento es posible siempre y cuando tenga una sombrilla que cubra estos procesos", y para ello está el horizonte institucional, del cual hacen parte los principios, los valores, los objetivos, la misión y la visión.

A esta acción se le llama, en el mundo de las organizaciones, direccionamiento estratégico institucional asunto que fue tenido en cuenta en el proceso de formación buscando de ésta forma empoderar a todos los miembros de la comunidad para que participen en un proyecto que enseña con el ejemplo. Porque en la medida que se vivan las actitudes emprendedoras y que se manifiesten en comportamientos, así mismo los estudiantes aprenderán a ser personas emprendedoras. (Tabla 5). 
Tabla 5. Enunciados de una gestión de la CIEE que empodera.

\begin{tabular}{|c|c|}
\hline Actor & Enunciado \\
\hline Dinamizadora regional & $\begin{array}{l}\text { La articulación de la CIEE significa responder a su quehacer } \\
\text { institucional con objetivos comunes que procuran el desarrollo } \\
\text { humano integral de los niños y los jóvenes, a través de actitudes } \\
\text { emprendedoras que fomentan comportamientos deseables y proyectos } \\
\text { de vida claros, lo que responde a las necesidades de los territorios. }\end{array}$ \\
\hline ENS La Candelaria & $\begin{array}{l}\text { El emprendimiento de tipo social trae a la luz y enuncia lo que } \\
\text { moviliza las prácticas de la institución formadora, dada su misión, su } \\
\text { visión y su tarea central, que es la formación de maestros, lo cual se } \\
\text { localiza en el campo del desarrollo humano y social. }\end{array}$ \\
\hline IE Santa Teresita & $\begin{array}{l}\text { El PEI, mediante el currículo afecta e incide en la instauración de una } \\
\text { CIEE pues pone el conocimiento en escena, lo reconstruye, lo válida } \\
\text { para armonizar las prácticas pedagógicas democráticas donde la } \\
\text { norma no solo es la excelencia, sino la renovación constante y atenta } \\
\text { de sus visiones frente a los imaginarios del contexto. }\end{array}$ \\
\hline IE San Francisco de Paula & $\begin{array}{l}\text { Los conflictos sociales que amenazan constantemente la escuela y } \\
\text { el potencial humano inquieto, ávido e inconforme, son en el insumo } \\
\text { principal para definir el énfasis del emprendimiento de la institución. }\end{array}$ \\
\hline IE San Francisco de Paula & $\begin{array}{l}\text { Planificar la propuesta pedagógica colaborativa que articule los } \\
\text { pensamientos, intenciones, estrategias de los actores intervinientes } \\
\text { requiere una planificación global que enmarque la finalidad de todos } \\
\text { hacia un objetivo común. }\end{array}$ \\
\hline IE San Francisco de Paula & $\begin{array}{l}\text { El concepto de emprendimiento social nace de la tensión entre una } \\
\text { concepción que ha instituido la dinámica empresarial, la lógica } \\
\text { de la productividad y el sentido de retribución económica, y una } \\
\text { concepción en la que prevalecen los deseos de resolver problemas } \\
\text { asociados a condiciones de exclusión, desigualdad y pobreza. } \\
\text { (Guzmán \& Trujillo, 2008) }\end{array}$ \\
\hline IE Intec & $\begin{array}{l}\text { El emprendimiento, una apuesta curricular en el INTEC, ha } \\
\text { trascendido todos los espacios de la institución, pues la trazabilidad } \\
\text { que permite y la manera como está concebida puede continuar } \\
\text { fortaleciendo la flexibilización como puerta siempre dispuesta } \\
\text { a permitir la entrada y la salida de situaciones que involucran la } \\
\text { institución en su política de mejora continua en todos los procesos. }\end{array}$ \\
\hline IE Marillac & $\begin{array}{l}\text { El Perfil de los integrantes de la comunidad educativa articulado a las } \\
\text { actitudes para el emprendimiento: } \\
\text { - Personas integrales } \\
\text { - Con calidad humana } \\
\text { - Con visón de futuro } \\
\text { - Eficientes } \\
\text { - Con comportamiento autorregulado } \\
\text { - Con capacidad de asumir riesgos } \\
\text { - Con capacidad de Identificar oportunidades y recursos del entorno } \\
\text { - Con creatividad y pensamiento flexible. }\end{array}$ \\
\hline
\end{tabular}




\begin{tabular}{|c|l|}
\hline \multicolumn{1}{|c|}{ Actor } & \multicolumn{1}{c|}{ Enunciado } \\
\hline IE Santa Teresita & $\begin{array}{l}\text { El proyecto del PRAE articulado a la propuesta de emprendimiento } \\
\text { Yo soy el flow y protejo el medio ambiente, donde los estudiantes } \\
\text { mostraron pancartas con mensajes de cambios positivos dando una } \\
\text { mirada fuerte al cuidado de su entorno, cuestionaban a sus pares para } \\
\text { que tomaran conciencia de lo que está pasando a su alrededor que } \\
\text { no sólo se quedará en palabras sino que es cuestión de compromiso } \\
\text { colectivo e individual }\end{array}$ \\
\hline Dinamizadora regional & $\begin{array}{l}\text { Las instituciones deben entender que hablar de cultura del } \\
\text { emprendimiento debe ser una apuesta, una filosofía, una nueva forma } \\
\text { de planeación y de articulación de los procesos institucionales que no } \\
\text { responsabiliza a un docente, por el contrario los debe comprometer a } \\
\text { todos los actores. }\end{array}$ \\
\hline
\end{tabular}

\section{De vuelta al sujeto}

Un dispositivo institucional según Giorgio Agamben en (Velásquez \& Zamudio, 2012, pág. 133) es un entramado que contiene un conjunto de relaciones, discursos, normas, decisiones que conforman una organización para ejercer el control, y que funcionan en las instituciones y están en consonancia con el ideal de sujeto que se quiere formar; sobre esto se resalta que las experiencias que han desarrollado procesos para fomentar la CIEE han generado unas elaboraciones propias institucionales que superan la visión empresarial, las cuales operan como dispositivos emergentes que enmarcan la concepción de una educación para el emprendimiento.

En esta línea, se encuentran ejercicios de sistematización que reivindican la formación de un sujeto emprendedor en la línea de un emprendimiento que responde a los intereses y necesidades institucionales y locales, y que de igual manera responden a los propósitos de la actual política pública de educación, cuyo eje de acción es afrontar los retos de una sociedad que después de 50 años asume la posibilidad de vivir en paz.

Tabla 6. Enunciados que resaltan la CIEE y el sujeto.

\begin{tabular}{|c|l|}
\hline Actor & \multicolumn{1}{c|}{ Enunciado } \\
\hline ENS La Candelaria & $\begin{array}{l}\text { De acuerdo con Cháux, Lleras y Velásquez (Chaux, 2004)“no se } \\
\text { duda del vínculo entre la formación ciudadana y la cultura del } \\
\text { emprendimiento, ya que es entendida por algunos autores como "los } \\
\text { conocimientos y habilidades que permiten que el ciudadano actúe de } \\
\text { manera constructiva en la sociedad democrática. }\end{array}$ \\
\hline IE San Francisco de Paula & $\begin{array}{l}\text { Las experiencias de los docentes en el proyecto de emprendimiento } \\
\text { social abrieron nuevas formas de pensar la práctica pedagógica } \\
\text { donde la creatividad y la innovación sedujeron principalmente a los } \\
\text { estudiantes y los docentes haciendo de este recurso la principal forma } \\
\text { de reproducción de las vivencias positivas y exitosas. }\end{array}$ \\
\hline
\end{tabular}




\begin{tabular}{|c|l|}
\hline \multicolumn{1}{|c|}{ Actor } & \multicolumn{1}{c|}{ Enunciado } \\
\hline IE San Francisco de Paula & $\begin{array}{l}\text { La dinámica que se incorporó en la IE permitió adoptar una } \\
\text { concepción que, en la práctica, se centra en la solución de } \\
\text { problemas de la vida escolar, acude al uso del método de árbol } \\
\text { de problemas, plantea, planifica soluciones y lleva a cabo un plan } \\
\text { para mejorar las situaciones que generan bienestar a la colectividad } \\
\text { social. }\end{array}$ \\
\hline ENS La Candelaria & $\begin{array}{l}\text { En la sistematización, no es posible eludir el papel que cumple } \\
\text { el área de gestión comunitaria con los procesos de extensión a } \\
\text { la comunidad, en la consolidación de la CIEE, puesto que se ha } \\
\text { incidido positivamente en la localidad y en la región, mediante } \\
\text { los proyectos que llevan a cabo mancomunadamente maestros y } \\
\text { estudiantes dirigidos a las comunidades; por ejemplo, el programa } \\
\text { de “lectura barrial”, los proyectos de intervención que benefician } \\
\text { a los niños y las niñas con necesidades de aprendizaje, a madres } \\
\text { gestantes, padres de familia a través de la escuela de padres, }\end{array}$ \\
\hline IE Intec & $\begin{array}{l}\text { El proyecto concluye que la necesidad del arte y la cultura en la } \\
\text { educación son cada vez más apremiantes. Encuentra entonces en } \\
\text { el emprendimiento artístico- cultural la oportunidad de descubrir, } \\
\text { despertar y motivar en los estudiantes y comunidad la sensibilidad } \\
\text { y el espíritu crítico que permite vivir una capacidad de asombro } \\
\text { y admiración por el mundo, estimula la capacidad de sortear } \\
\text { situaciones y fortalece un proyecto de vida }\end{array}$ \\
\hline IE Intec & $\begin{array}{l}\text { La institucionalización de las actitudes emprendedoras como } \\
\text { pilares pedagógicos que fundamentan el desarrollo de las prácticas } \\
\text { pedagógicas han fortalecido actitudes y aptitudes en los estudiantes } \\
\text { como cimientos del proyecto de vida que la institución anhela } \\
\text { inspirar en los niños, los adolescentes y los jóvenes que adquieren la } \\
\text { esperanza al transitar por las aulas. } \\
\text { El trabajo intencionado a crear actitudes emprendedoras } \\
\text { ha trascendido hasta las familias, pues se hacen encuentros } \\
\text { familiares actúa y emprende en familia, para crear lazos afectivos } \\
\text { que estimulen la corresponsabilidad como un componente de } \\
\text { flexibilización curricular. }\end{array}$ \\
\hline
\end{tabular}

\section{Discusión}

A partir del análisis de los enunciados se encuentran elementos de reflexión en torno a dos líneas: frente al proceso y logros institucionales y el aporte a la política pública de educación.

\section{Frente al proceso y los logros}

En particular en la macro categoría "Las prácticas que movilizan" se identifica en las experiencias de algunas instituciones que los principales aspectos para generar una CIEE son: Un direccionamiento estratégico que orienta la gestión institucional y moviliza el compromiso de la comunidad educativa llevando a tomar decisiones 
consensuadas y realizar un análisis colectivo sobre una educación para el emprendimiento de alto impacto, que responda a los retos que el mundo social y productivo propone actualmente y coherente con las necesidades del entorno.

Las acciones para el fomento de la CIEE indican que las instituciones son entes vivos, con unos ideales que estimulan las prácticas que tengan sentido, para formar personas integrales con actitudes emprendedoras que los lleve a convivir en paz desarrollarse y crecer de manera integral.

Se encuentra una estrecha relación entre lo que ha significado la CIEE y el fortalecimiento de la gestión, en la reorganización de procesos institucionales, entre ellos, la toma de decisiones frente al desmonte de la cátedra de emprendimiento y la optimización del proceso educativo en el diseño y desarrollo del currículo con un enfoque nuevo, donde se evidencia la articulación y transversalización de las actitudes emprendedoras en las diversas áreas de formación y proyectos pedagógicos institucional.

En relación con la macro categoría "cambio de paradigma", se evidenció que este fue producto de un cambio en la dinámica institucional que partió de la apropiación de un discurso sobre una educación para el emprendimiento que trasciende el enfoque empresarial y se centra en el desarrollo de actitudes, que moviliza acciones articuladas al horizonte institucional, donde se lograron vincular todos los diversos actores de la comunidad educativa, en busca de coherencia, pertinencia proyección y sostenibilidad .

Cabe resaltar que éste proceso fue producto de la cualificación dada a las instituciones donde a sus maestros se les dio lugar especial en el cual se pudieron expresar las ideas, movilizar cambios que les permitieron reconocer lo que son y para dónde van en el contexto de la CIEE. Es aquí donde la experiencia permitió darle un lugar al sujeto maestro, sujeto estudiante, sujeto directivo y padre de familia como actores fundamentales para generar una cultura institucional que propicie el aprendizaje en la práctica diaria.

\section{Frente al aporte a la política pública de educación}

Para determinar los aportes que estas experiencias brindan a la política pública se hace necesario partir de lo que ésta significa y las características de la agenda nacional que define la política educativa actual para así comprender las categorías de análisis iniciales trabajadas.

De acuerdo con Yepes, las políticas públicas son:

(...) un dispositivo con el cual cuenta un gobierno para el ejercicio de la misión que la sociedad le ha encomendado, en el cual se refleja la cosmovisión de quienes detentan el poder político y condensan las acciones del estado, a través de sus instituciones y organizaciones para intervenir sobre situaciones socialmente problemáticas o para evitar hechos que alteren el statu quo 2016, pág. 49

En relación con esta perspectiva, (Yepes, 2016) afirma que las políticas que se enriquecen con una dinámica participativa permiten que la acción gubernamental responda a situaciones que requieren su intervención.

En este sentido, señala la importancia de escuchar a otros actores con el fin de establecer políticas públicas en educación que abandonen una visión hegemónica del 
poder y promuevan una nueva relación estado-sociedad donde la sociedad tenga un papel protagónico.

Por ésta razón llamamos la atención para que las voces de las instituciones y de los maestros y maestras participantes en éste proyecto sean escuchadas y analizadas y de esta manera aporten a la concepción de una educación para el emprendimiento pertinente para los territorios y sus comunidades. Para comprender esta situación, conviene resaltar que las instituciones se han enfrentado a varios discursos gubernamentales que han planteado distintos enfoques que se señalaban al comienzo de este documento, y que se caracterizan por intereses de poder que buscan introducir el tema, desde un enfoque productivo en la educación preescolar, básica y media. Como consecuencia del proceso de formación y el ejercicio de sistematización, han surgido nuevos conocimientos que indican el empoderamiento de un discurso institucional que reconfigura lo establecido en algunos documentos gubernamentales, con concepciones más contextualizadas y pertinentes.

Tal como lo señala el estudio de la política pública de emprendimiento en Colombia desarrollado por (Tarapuez, Osorio, \& Botero, 2013, pág. 282) en el que exponen la necesidad de articular una concepción de emprendimiento con visión y prospectiva que permita dar la respuesta a problemas sociales con ideas emprendedoras, asunto que se demuestra en la línea de trabajo emprendida por las instituciones que participaron en este estudio y que en cierta forma se convierte en un insumo valioso para el desarrollo social y económico de las regiones.

Según estos referentes, es conveniente incluir el emprendimiento como un punto estratégico de desarrollo en Colombia, partiendo de lo que ha establecido el Ministerio de Educación Nacional y complementado con lo que han venido haciendo las instituciones en esta línea, en este sentido, los elementos que se deben tener en cuenta para enriquecer la política pública de educación son:

\section{Direccionamiento de la política pública a partir de la CIEE}

Las políticas educativas se plantean con el propósito de producir respuestas a problemáticas o retos coyunturales, actualmente Colombia enfrenta el desafío de una educación para el posconflicto, donde la CIEE se convierte en una oportunidad para desarrollar procesos educativos innovadores que respondan a las demandas sociales y productivas .En este sentido, las experiencias sistematizadas aportan una ruta para que la CIEE sea un referente que propicie transformaciones de fondo en la planeación educativa y en la proyección a la comunidad.

Por esta razón, es recomendable que la política pública educativa retome elementos propios de los proyectos educativos regionales donde el emprendimiento tiene un lugar importante

\section{La calidad desde una perspectiva de desarrollo humano}

La medición de la calidad educativa se ha centrado en los resultados de pruebas externas y estandarizadas, una educación orientada al fomento de la CIEE permite valorar la calidad, desde una educación pertinente para los territorios que responda a intereses y necesidades buscando transformar las formas de pensar el emprendimiento, enriqueciendo prácticas curriculares para movilizar proyectos de vida en función del desarrollo personal y social. 


\section{Sinergias en la CIEE}

La CIEE invita a la conformación de equipos interdisciplinarios que adelanten un trabajo colaborativo, que permite a las instituciones el desarrollo de acciones de mejoramiento y empoderamiento, lo que es vital en una política pública que busque fortalecer la educación en las regiones, se llama la atención para que promueva la articulación con redes y organizaciones que no solo apoyen emprendimientos de tipo empresarial, como lo plantea que la ley 1014 de 2006 sino hacia otros tipos de emprendimientos tales como, cultural, social, ambiental, tecnológicos, entre otros, que se han hecho evidentes en el contexto de las instituciones que participaron en el proceso, de tal manera que se atienda a las diversas a necesidades e intereses del entorno.

\section{Acompañamiento a las instituciones educativas}

El éxito en la implementación de una política educativa, no solo depende de su promulgación, sino sobre todo del acompañamiento que se haga en las instituciones educativas para su interpretación e implementación. Las experiencias sistematizadas evidenciaron como esto fue factor clave para que la CIEE se articulara al currículo garantizando proyección y sostenibilidad.

\section{El sujeto como centro de la política}

Una política educativa que plantee la formación de personas emprendedoras se debe orientar hacia la construcción de proyecto de vida y no solo a la solución de problemas sociales coyunturales, como el desempleo, esto implica reconocer a las personas y comunidades en todas sus dimensiones y orientar acciones que promuevan el desarrollo humano integral.

\section{Conclusiones}

Las experiencias sistematizadas aportan saberes y conocimientos valiosos que muestran como la formación para el emprendimiento desde una perspectiva integral, que trasciende la empresarialidad, es una oportunidad de construir proyectos educativos pertinentes con propuestas curriculares coherentes y articuladas que permiten movilizar voluntades para un propósito común.

En este sentido, un país como Colombia que está en un momento de transición y cierre de brechas, debería pensar que las políticas educativas se deben formular en consonancia con la experiencia de maestros y las instituciones educativas, ya que esto permite que se orienten proyectos y estrategias coherentes con las realidades, necesidades y oportunidades manifiestas en las comunidades.

Realizar procesos de sistematización de experiencias constituye una estrategia clave para que los diversos actores que participan en los procesos educativos reconozcan y se empoderen de saberes y experiencias que permiten desarrollar procesos de participación en la construcción de políticas educativas.

Los esfuerzos que realizan las instituciones educativas para llevar a la práctica las políticas públicas educativas son aprendizajes valiosos, prácticas pedagógicas 
innovadoras, que se deben reconocer e incluir en la agenda pública como un ejemplo orientador que permita conseguir las metas planteadas y que incluya una comprensión diferente de la educación para el emprendimiento en estos tiempos.

\section{Agradecimientos}

Agradecemos a la Chec-Grupo epm, en especial al Dr. Gonzalo Jaramillo Hoyos y a Diego Duque Escobar; a las Secretarias de Educación de Caldas en especial a la Dra. Martha Gloria Peláez, y las Secretarias de Risaralda y Manizales; a la Universidad de Manizales; a los establecimientos educativos participantes; a los dinamizadores regionales Cesar Castaño y Gloria Grajales y por último a Unicafam, a las directivas, en cabeza del Dr. Francisco Cajiao (Rector), la Dra. Maritza Cristancho García (Vicerrectora Académica) y al Dr. Mikhail Benet (Director de Investigaciones); así como a todas las personas y profesionales que ofrecieron su tiempo para compartir ideas y seguir empoderando al magisterio colombiano.

\section{Referencias Bibliográficas}

Chaux, E. (2004). Introducción. En Competencias Ciudadanas: De los Estándares al Aula: Una propuesta de integración a las áreas académicas. (pp. 13-25). Bogotá: Ministerio de educación Nacional.

Dallorso, N. (Mayo de 2012). Notas sobre el uso del concepto de dispositivo para el análisis de programas sociales. Espiral, 19(54), 43-74. Recuperado el 4 de Mayo de 2017, de Academic search complete.

Plan nacional de desarrollo 2014-2018. Presidencia de la república de Colombia. (2014). Recuperado de https://colaboracion.dnp.gov.co/CDT/PND/PND\%202014-2018\%20 Tomo\%201\%20internet.pdf

Foucault, M. (2004). Arqueología del saber. Buenos Aires: Siglo XXI.

Guzmán, A., \& Trujillo, M. A. (2008). Emprendimiento social: Revision de la literatura. Estudios gerenciales, 24(109), 105-125.

Ley 1014. De fomento a la cultura del emprendimiento. Diario oficial 46164. Congreso de Colombia (26 de enero de 2006).

Mejía, M. R. (Mayo de 2012). La sistematización una forma de investigar las prácticas y producir saber y conocimiento. En V. d. Especial (Ed.), Calidad Educativa y Sistematización. Quito: Fe y Alegría - Ecuador.

Ministerio de Educación Nacional. (2006). Articulación de la educación con el mundo productivo: competencias laborales generales. Serie guías No. 21. Bogotá: Autor.

Ministerio de Educación Nacional. (2012). La cultura del emprendimiento en los establecimientos educativos: orientaciones generales. Serie guías No. 21. Bogotá:

Ministerio de Educación, Cultura y Deporte. (2015). La educación para el emprendimiento en el sistema educativo español: Año 2015. [Madrid, España] Recuperado de: http:// www.sel-gipes.com/uploads/1/2/3/3/12332890/2016_-_ue_-_entrepreneurship_education_at_school_in_europe_sp.pdf

Molina, S. (2001). La investigación de segundo orden en ciencias sociales y su potencial predictivo: el caso del proyecto de Identidad y tolerancia. Revista Mexicana de Ciencias Políticas y Sociales, XLIV (183), 17-46 
Oliver, A., Galiana, L., y Gutiérrez, M. (2016). Diagnóstico y políticas de promoción del emprendimiento en los estudiantes. Anales de Psicología, 32(1).

Sampieri, R., Fernández, C., \& Baptista, P. (2010). Metodología de la investigación. México: Mc Graw Hill.

Sánchez, J. (2006). Los retos de lo local en lo global: aportes analíticos y normativos. Iconos, $25,77-88$.

Tarapuez, E., Osorio, H., y Botero, J. J. (2013). Política de emprendimiento en Colombia 2002-2010. Estudios Gerenciales, 29 (128).

Velásquez, J. y Zamudio, H. (2012). La articulación de la educación media con la educación superior, su análisis a partir del concepto de dispositivo. Aletheia, 4(2), 130-149.

Yepes, J. (2016). Retos y dilemas de la educación superior pública en Colombia. Manizales: Universidad de Caldas. 